\title{
Cardiac Magnetic Resonance Imaging in Polyarteritis Nodosa
}

HITOMI KOBAYASHI, MD, PhD; ISAMU YOKOE, MD, Division of Rheumatology, Itabashi Chuo Medical Center, Tokyo; NAOICHIRO HATTAN, MD; HIROSHI OHTA, MD, Division of Cardiology, Itabashi Chuo Medical Center; YASUO NAKAJIMA, MD; YASUYUKI KOBAYASHI, MD, Department of Radiology, St. Marianna University School of Medicine, Kawasaki, Kanagawa, Japan. Address correspondence to Dr. H. Kobayashi; E-mail: haraoka@pero.name. J Rheumatol 2010;37:11; 2427-9; doi:10.3899/jrheum.100450

Although cases of coronary artery aneurysm formation and myocardial infarction due to dissection in patients with polyarteritis nodosa (PAN) have been reported ${ }^{1,2}$, there are no reports evaluating widespread coronary arteritis accompanied by myocardial damage using cardiac magnetic resonance imaging (cMRI). We performed cMRI on a young man with widespread coronary arteritis resulting from PAN, and evaluated the changes in myocardial involvement after 3 months.

This case involved a 35-year-old male who, in July 2009, noted unintentional weight loss (4 kg in 1 month), fever, and joint pain. Toward the end of August, he presented for examination after noticing paresthesia and myalgia in both lower extremities and discomfort in the chest unrelated to physical exertion. There was nothing remarkable in medical histories of the patient or family. There were no irregular vital signs but there was muscular tenderness in both lower extremities. Electrocardiogram revealed slightly elevated ST in II, III, and aVF leads. Laboratory examination showed leukocytosis $(11,000 / \mu \mathrm{l})$, elevated C-reactive protein $(11.42 \mathrm{mg} / \mathrm{dl})$, and elevated creatine kinase (CPK 170 U/1, CPK-MB 35.9), but no evidence of renal or hepatic dysfunction. No obvious infectious foci could be identified. Tests for antinuclear antibody, HBsAg, hepatitis $\mathrm{C}$ virus antibody, syphilis rapid plasma reagin, cryoglobulin, and immune complex were negative, and myeloperoxidase-antineutrophil cytoplasmic antibodies (ANCA) and proteinase 3-ANCA levels were $<10$ $\mathrm{EU} / \mathrm{ml}$. A chest radiograph revealed no abnormalities. Echocardiography examination revealed posterior-wall and inferior-wall hypokinesis, and ejection fraction was $63 \%$. On cMRI, a delayed-enhanced (DE) image showed linear enhancements in the subendocardial layer, suggesting myocardial infarctions (Figure 1). A coronary angiography examination showed widespread stenosis in multiple peripheral blood vessels and vascular wall abnormalities, and the patient was diagnosed as having coronary arteritis (Figure 2). He was also diagnosed with PAN based on paresthesia and myalgia in bilateral lower extremities, weight loss, and angiography findings. Moreover, 3 of the American College of Rheumatology diagnostic criteria ${ }^{3}$ for PAN were confirmed. He was started on $60 \mathrm{mg}$ prednisone and $100 \mathrm{mg}$ cyclophosphamide orally per day. Inflammatory response was suppressed and the symptoms disappeared.

An ensuing electrocardiogram showed T-wave inversions

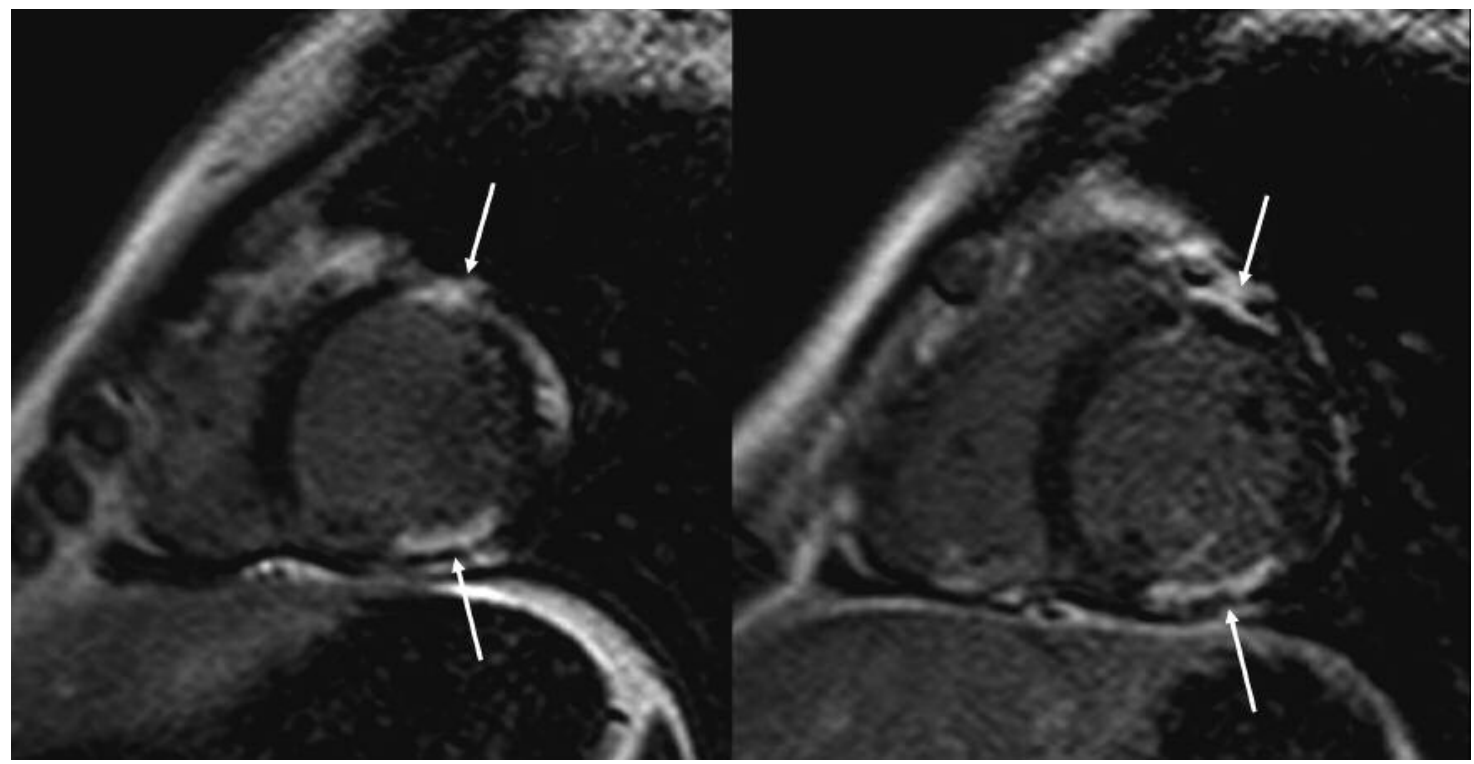

Figure 1. Delayed-enhanced cardiac MRI showed linear enhancements in mainly subendocardial layer of anterior and inferior wall (arrows), suggesting myocardial infarctions.

Personal non-commercial use only. The Journal of Rheumatology Copyright @ 2010 . All rights reserved. 
in II, III, and aVF leads. However, in a cMRI after 3 months, high signal intensity, indicating edema, was shown on the "black-blood" T2-weighted inversion-recovery MRI (Figure 3A). A stress perfusion MRI (Figure 3B) showed nonseg- mental circumferential perfusion defects (arrows) suggesting microvascular impairment. The DE image (Figure 3, C1, C2) showed multiple curvilinear enhancements with border irregularities in the subendocardial and middle layers, which

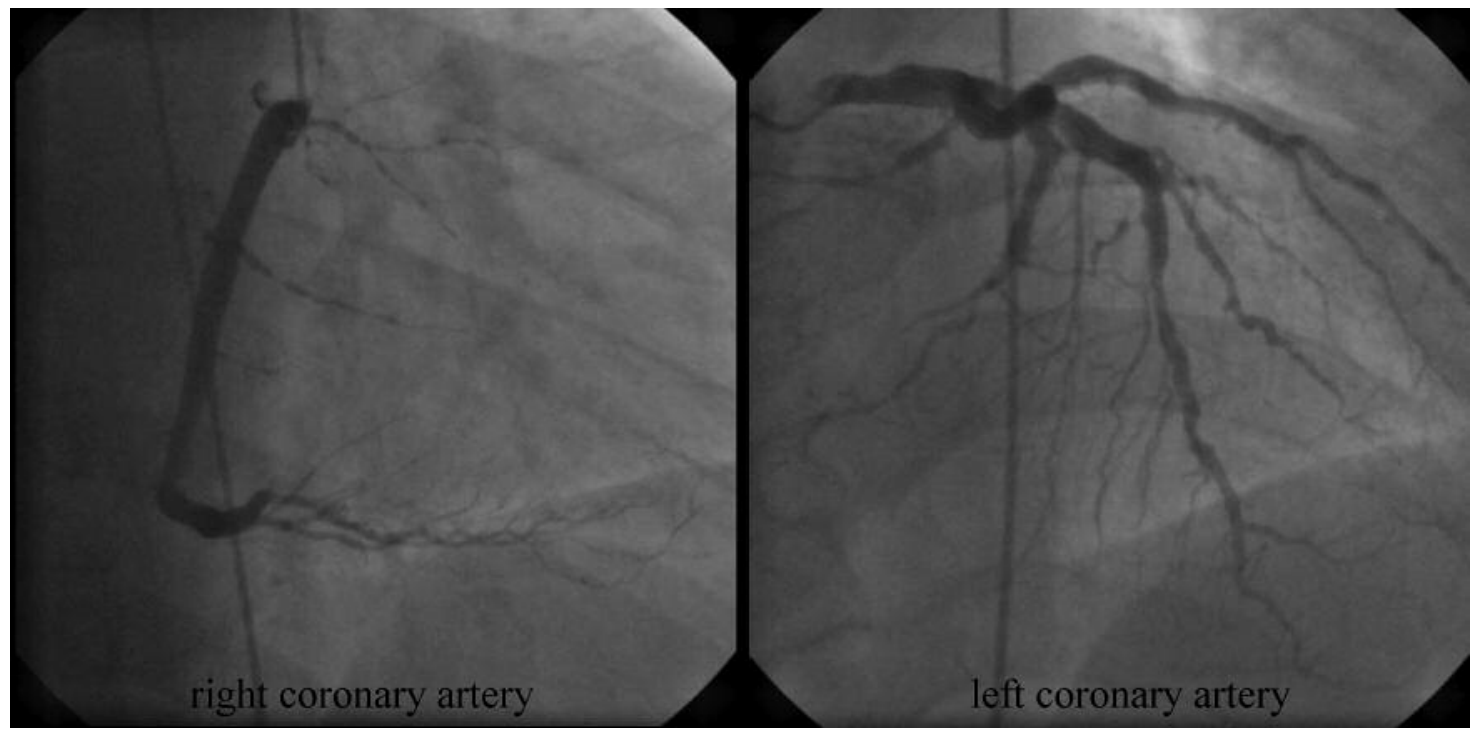

Figure 2. Coronary angiogram of right and left coronary arteries. In the distal branches of all coronary arteries, diffuse lesions with corkscrew-like appearance were present. These findings were compatible with coronary arteritis.

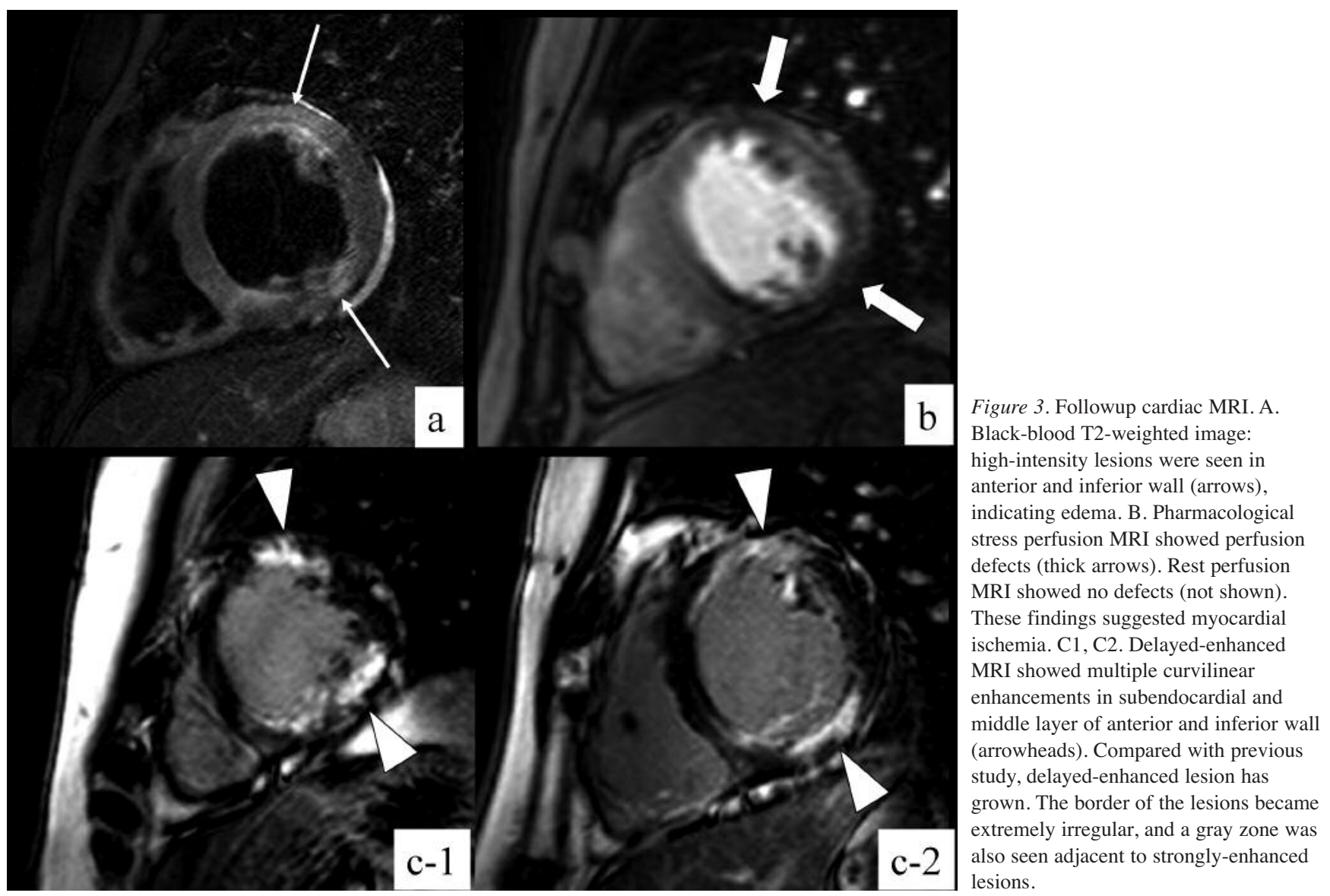

Personal non-commercial use only. The Journal of Rheumatology Copyright @ 2010 . All rights reserved. 
had increased in size. A gray zone was also observed adjacent to the strongly enhanced lesions. These findings of cMRI suggest the possibility of progression in myocardial involvement, regardless of PAN activity. In terms of PAN, despite alleviating the observed inflammation and dissipation of symptoms, cardiac involvement may progress, an important consideration to keep in mind.

Cardiac MRI can be useful as a far less invasive method of evaluating myocardial damage in patients with PAN.

\section{REFERENCES}

1. Maillard-Lefebvre H, Launay D, Mouquet F, Gaxotte V, Hachulla E, de Groote P, et al. Polyarteritis nodosa-related coronary aneurysms. J Rheumatol 2008;35:933-4.

2. Chu KH, Menapace FJ, Blankenship JC, Hausch R, Harrington T. Polyarteritis nodosa presenting as acute myocardial infarction with coronary dissection. Cathet Cardiovasc Diagn 1998;44:320-4.

3. Lightfoot RW Jr, Michel BA, Bloch DA, Hunder GG, Zvaifler NJ, McShane DJ, et al. The American College of Rheumatology 1990 criteria for the classification of polyarteritis nodosa. Arthritis Rheum 1990;33:1088-93. 